Rev. Bras. Saúde Prod. Anim., Salvador, v.14, n.3, p.599-608 jul./set., 2013 http://www.rbspa.ufba.br ISSN 15199940

\title{
Interação genótipo-ambiente em rebanhos nelore da Amazônia Legal
}

\author{
Genotype by environment interaction in Nellore cattle from Amazon Legal region
}

\author{
MATOS, Amanda de Sousa ${ }^{1 *}$; SENA, Josynélia do Socorro da Silva ${ }^{1}$; MARCONDES, \\ Cintia Righetti ${ }^{2}$; BEZERRA, Luiz Antonio Framartino ${ }^{3}$; LÔBO, Raysildo Barbosa ${ }^{4}$; \\ RORATO, Paulo Roberto Nogara ${ }^{5}$; CUCCO, Diego de Córdova ${ }^{6}$; ARAÚJO, Ronyere \\ Olegário de ${ }^{7}$
}

\footnotetext{
${ }^{1}$ Universidade Federal do Pará, Núcleo de Ciências Agrárias e Desenvolvimento Rural, Programa de PósGraduação em Ciência Animal, Belém, Pará, Brasil.

${ }^{2}$ Embrapa Pecuária Sudeste, São Carlos, São Paulo, Brasil

${ }^{3}$ Universidade de São Paulo, Faculdade de Medicina de Ribeirão Preto, Ribeirão Preto, São Paulo, Brasil.

${ }^{4}$ Associação Nacional de Criadores e Pesquisadores, Ribeirão Preto, São Paulo, Brasil.

${ }^{5}$ Universidade Federal de Santa Maria, Departamento de Zootecnia, Santa Maria, Rio Grande do Sul, Brasil.

${ }^{6}$ Universidade do Estado de Santa Catarina, Centro de Educação Superior do Oeste, Chapecó, Santa Catarina, Brasil.

${ }^{7}$ Universidade de Brasília, Campus Universitário Darcy Ribeiro, Brasília, Distrito Federal, Brasil.

*Endereço para correspondência: amanda_smatos@hotmail.com
}

\section{RESUMO}

A interação genótipo-ambiente (IGA) foi estudada por meio de dois procedimentos distintos, em características produtivas e reprodutivas de bovinos da raça Nelore. Dados de pesos padronizados aos 120 (P120); 210 (P210); 450 (P450) dias de idade, perímetro escrotal aos 450 dias de idade (PE450) e idade ao primeiro parto (IPP), de 211.744 registros de animais Nelore, criados na região da Amazônia Legal, foram utilizados na análise. O efeito da IGA foi estudado por meio de estimativas de herdabilidade e de correlações entre classificações, comparando os animais da Amazônia Legal com a base geral de animais do PMGRN - Nelore Brasil. As análises bi-característica consideraram o P120 como característica-âncora, com P210; P450 e PE450. A característica IPP foi analisada separadamente, em análise de característica única e considerando como efeito fixo o GCIPP e como aleatórios os efeitos aditivos genético e residual. As estimativas de herdabilidade para P120; P210; P450; PE450 e IPP nos dados da Amazônia Legal foram: 0,20 a 0,49; 0,21;0,48; 0,45 e 0,21 , respectivamente, e nos dados gerais do PMGRN - Nelore Brasil foram: 0,23; 0,25; 0,$34 ; 0,43$ e 0,11 , respectivamente. As correlações entre classificações de rank para P120; P210; P450; PE450 e IPP foram iguais a
0,$77 ; 0,79 ; 0,82 ; 0,78$ e 0,38 , respectivamente. As análises da IGA, por meio das estimativas de herdabilidade, evidenciaram maiores efeitos sobre os aspectos maternos, de peso aos 450 dias de idade e idade ao primeiro parto, enquanto que as correlações entre classificações mostraram fortes evidências em quase todas as características estudadas.

Palavras-chave: bovinos, correlação de rank, modelo animal, parâmetros genéticos

\section{SUMMARY}

Genotype-environment interaction was studied by two different procedures in productive and reproductive traits of Nellore cattle. Data from adjusted weights at 120 (P120), 210 (P210), 450 (P450) days of age, scrotal circumference at 450 days of age (PE450) and age at first calving (IPP) for 211,744 records from Nellore herds located in the Legal Amazon region were used in the analysis. The effect of genotypeenvironment interaction was studied through heritability estimates and rank correlation, comparing the Legal Amazon animals with the general basis of animals - PMGRN Nellore Brazil. Bi-trait analyses considered P120 as anchor-trait with P210, P450 and PE450 as 
another one. IPP has been analyzed separately in single-trait analysis and considering GCIPP as fixed and additive and residual effects as random. Estimates of heritability for P120, P210, P450, PE450 and IPP on the data of the Legal Amazon were: 0.20 to $0.49 ; 0.21 ; 0.48$; 0.45 ; and 0.21 , respectively, and in general data of PMGRN - Nellore Brazil were: 0.23; 0.25; $0.34 ; 0.43$ and 0.11 , respectively. Correlations between rank for P120, P210, P450, PE450 and IPP were equal to $0.77 ; 0.79 ; 0.82 ; 0.78$ and 0.38 , respectively. The analysis of the genotypeenvironment interaction, through the heritability estimates, showed larger effects on maternal, weight at 450 days of age and age at first calving, whereas the rank correlations showed strong evidence in almost all traits studied.

Keywords: Beef cattle; rank correlation; animal model; genetic parameters

\section{INTRODUÇÃO}

Animais melhoradores participantes de programas de seleção obedecem a uma ordem de classificação de acordo com o seu desempenho. Esta ordem pode ser modificada quando se desconsidera a presença da interação genótipoambiente (IGA). A importância da IGA vem crescendo nos últimos anos devido à globalização das criações e do melhoramento animal (KOLMODIN \& BIJMA, 2004), tornando-se um fator de relevância quando se seleciona animais considerados superiores. Baseado no fato de que a IGA pode prejudicar o progresso genético das populações de bovinos de corte pelo uso inadequado de reprodutores, é de fundamental importância a consideração desta interação nas avaliações genéticas.

Em 1953, através da Lei 1.806, de 06/01/1953, a Amazônia Brasileira passou a ser chamada de Amazônia Legal, fruto de um conceito político e não de um imperativo geográfico. Atualmente, os Estados que compõe a Amazônia Legal são: Acre, Amapá, Amazonas, Mato Grosso, Pará,
Rondônia, Roraima, Tocantins e parte do Maranhão (Oeste do meridiano de $44^{\circ}$ ), perfazendo uma superfície de aproximadamente $\quad 5.217 .423 \quad \mathrm{~km}^{2}$ correspondente a cerca de $60 \%$ do território brasileiro (SUDAM, 2013). Segundo o IBGE (2009), o Brasil conta com 172.613.337 de cabeças de bovinos, sendo a Amazônia Legal responsável por $31,6 \%$ deste valor. A expansão da fronteira agrícola, a implantação de frigoríficos e de rotas comerciais de exportação da carne e de animais vivos têm aumentado, substancialmente, o investimento na bovinocultura comercial da região.

A IGA pode ser detectada pela estimação de componentes de variância, comparando-se modelos que incluam ou não o efeito de interação (DIMOV et al., 1995), ou ainda pela comparação entre as classificações dos animais, caso sejam significativas, de acordo com os valores genéticos preditos, por meio dos vários modelos existentes (BASU \& CHATTARAJI, 1988; TORAL et al., 2004; RIBEIRO et al., 2009). Mais recentemente, análise Bayesiana (FALCÃO et al., 2006; SOLAR DIAZ et al, 2011) e normas de reação via modelos de regressão aleatória (CORRÊA et al., 2009) têm sido empregadas nos estudos de IGA.

Neste estudo foram aplicadas análises para estimativas de parâmetros genéticos nos rebanhos da Amazônia Legal e na base de dados do PMGRN - Nelore Brasil, considerando as características como distintas e correlacionando-as, posteriormente, com o objetivo de testar a presença da interação genótipo-ambiente em características produtivas $\mathrm{e}$ reprodutivas de bovinos da raça Nelore. 


\section{MATERIAL E MÉTODOS}

$\mathrm{O}$ arquivo de dados analisado consistia em 211.744 registros de animais da raça Nelore, participantes do Programa de Melhoramento Genético da Raça Nelore (PMGRN - Nelore Brasil), nascidos no período de 1995 e 2008, criados em regime de pasto e distribuídos em 44 rebanhos localizados nos seguintes Estados: Acre (AC), Maranhão (MA), Mato Grosso (MT), Pará (PA), Rondônia (RO) e Tocantins (TO). Todos os animais foram monitorados do nascimento aos 21 meses de idade e as matrizes também pesadas ao parto, na desmama dos seus produtos e nos meses de abril e outubro de cada ano.

O clima das fazendas participantes do Programa é característico da região Amazônica brasileira, caracterizado por duas estações, sendo uma seca (maio a setembro) e outra chuvosa (outubro a abril), com regime pluviométrico médio anual, normalmente entre $1.250 \mathrm{~mm} \mathrm{e}$ $2.500 \mathrm{~mm}$, decrescendo de sudoeste para nordeste, estando sujeito a importantes flutuações. Sob a influência da baixa latitude, as temperaturas mantêm-se elevadas durante todos os meses do ano e suas médias térmicas anuais são superiores a $22^{\circ} \mathrm{C}$ nas partes mais elevadas, setores norte e oeste, aumentando de norte para sul até $27^{\circ} \mathrm{C}$, quando se aproxima do Equador, com pequena amplitude térmica anual. Devido a fatores geográficos (latitude e relevo) e a fatores dinâmicos, esta região possui altos níveis de eficiência térmica durante todo o ano, razão pela qual é caracterizada por clima Megatérmico, no setor oeste, e em parte do norte (altitude de mais de $800 \mathrm{~m}$ ) o clima é Mesotérmico, tendendo para o Megatérmico. A umidade relativa do ar, média anual, situa-se entre $60 \%$ e $85 \%$, aumentando da zona setentrional para a meridional (SUDAM, 2013).

O PMGRN - Nelore Brasil padroniza os pesos à determinada idade-padrão (120 dias; 205 dias; 365 dias; 450 dias ou 550 dias), necessitando-se para isso que cada animal tenha uma pesagem anterior e uma pesagem posterior a esta data. A seguinte fórmula foi utilizada para a obtenção do peso à idade-padrão: Peso à idade-padrão $=\mathrm{Pa}+(\mathrm{GMD} \mathrm{x}$ da), em que $\mathrm{Pa}$ é peso anterior à idadepadrão, GMD é o ganho de peso médio diário e da são os dias compreendidos entre a pesagem anterior e a idadepadrão.

Cada animal possui uma identificação única e permanente, com registro dos pais, número da fazenda de origem (NFO) e atual (NFA), Estado (UF), sexo (SX), ano (ANO) e mês (MÊS) de nascimento, classe de idade da vaca ao parto (CIVP), peso padronizado aos $120 ; 210$ e 450 dias (P120; P210 e $\mathrm{P} 450$ ), perímetro escrotal padronizado aos 450 dias (PE450), idade ao primeiro parto (IPP), lote aos $120 ; 210$ e 450 dias (LOTE120; LOTE210 e LOTE450), grupo contemporâneo (GC) aos 120; 210 e 450 dias (GC120; GC210 e GC450) e grupo contemporâneo para IPP (GCIPP). Os grupos contemporâneos contêm os efeitos de fazenda, sexo, estação de nascimento e lote de manejo à idade padrão, sendo que o GC450, por exemplo, concatena os efeitos dos lotes de manejo aos 120, aos 210 e aos 450 dias de idade. O GCIPP contempla efeitos de fazenda e lote de manejo aos 550 dias de idade.

A consistência dos dados, as análises descritivas e de correlação de rank foram realizadas utilizando-se o software Statistical Analysis System (SAS Intitute, 2002). Medidas iguais a zero ou acima/abaixo de três desvios-padrão não foram utilizadas nas análises, bem como 
grupos de contemporâneos (GC450) com menos de sete animais.

Os componentes de (co)variância atribuídos a cada efeito aleatório foram estimados por intermédio do programa MTDFREML (BOLDMAN et al., 1995). As análises bicaracterística consideraram o P120 como característica-âncora, com P210; P450 e PE450. A característica IPP foi analisada separadamente, em análise de unicaracterística e considerando como efeito fixo o GCIPP e como aleatórios os efeitos genéticos aditivos e residuais. $\mathrm{O}$ modelo animal utilizado na análise conjunta de P120 e P210 considerou, além dos efeitos fixos de grupo contemporâneo (GC120 e GC210, respectivamente) e de CIVP, os efeitos aleatórios genéticos aditivos, maternos e residuais. Nas análises de P120 com P450 e com PE450 foi desconsiderado o efeito materno no modelo. Não foi considerado na análise para as características pré-desmama o efeito de ambiente permanente da vaca, pois a média de progênies/vaca foi inferior a dois. A covariância aditivo-materna foi fixada em zero, conforme protocolo estabelecido nas análises do PMGRN Nelore Brasil.

Em termos matriciais, os modelos com e sem efeito materno pode ser descritos como:

$\mathrm{y}=\mathrm{Xb}+\mathrm{Z1a}+\mathrm{Z} 2 \mathrm{~m}+\mathrm{e}$

em que, $\mathrm{y}=$ vetor de observações; $\mathrm{b}=$ vetor dos efeitos fixos; $a=$ vetor de efeitos genéticos aditivo direto; $\mathrm{m}=$ vetor dos efeitos genéticos aditivo materno (presente somente nas análises de P120 e P210); e e = vetor dos erros aleatórios residuais associados às observações. X, Z1 e Z2 são matrizes de incidência para cada efeito.

$y=\mathrm{X} b+\mathrm{Z} 1 a+e$

em que, $\mathrm{y}=$ vetor de observações; $\mathrm{b}=$ vetor dos efeitos fixos; $a=$ vetor de efeitos genéticos aditivos direto e e $=$ vetor dos erros aleatórios residuais associados às observações. $\mathrm{X}$ e $\mathrm{Z1}$ são matrizes de incidência para cada efeito.

As pressuposições assumidas pelos modelos foram: $\mathrm{E}(\mathrm{y})=\mathrm{Xb} ; \mathrm{E}(\mathrm{a})=0$; $\mathrm{E}(\mathrm{m})=0 ; \mathrm{Va}(\mathrm{a})=\mathrm{A} \sigma^{2} \mathrm{a} ; \mathrm{Va}(\mathrm{m})=$ $\mathrm{A} \sigma^{2} \mathrm{~m} ; \mathrm{Va}(\mathrm{e})=\mathrm{I}_{\mathrm{n}} \sigma^{2} \mathrm{e}$, em que A é a matriz de parentesco, $\sigma^{2}$ a é a variância genética aditiva, $\sigma^{2}$ e é a variância residual e I é uma matriz Identidade de ordem $r$ por $r$.

O critério de convergência utilizado, definido pelo quadrado médio das diferenças entre soluções consecutivas, foi de $1 \times 10^{-9}$, com diferença entre análises sucessivas na quarta casa do 2LogL.

A matriz de parentesco continha 73.272 animais.

As Diferenças Esperadas na Progênie (DEPs) dos animais foram obtidas por meio da saída MTDF78, a qual contém as soluções para cada animal. As DEPs da base geral de dados do PMGRN Nelore Brasil, para os 73.272 animais, foram solicitadas à Associação Nacional de Criadores e Pesquisadores - ANCP, para realizar a comparação entre classificações, por meio do procedimento PROC CORR opção Spearman do SAS. $\mathrm{O}$ arquivo de dados inicial continha 1.328 touros com média de 16 progênies/touro. O maior percentual $(87 \%)$ dos touros foi utilizado por até quatro anos e os grupos contemporâneos continham entre cinco e 15 touros.

\section{RESULTADOS E DISCUSSÃO}

As médias para P120; P210 e P450 (Tabela 1) apresentaram resultado similar à média do PMGRN - Nelore Brasil, de $125 \mathrm{~kg} ; 183 \mathrm{~kg}$ e $272 \mathrm{~kg}$ respectivamente para as mesmas características (LÔBO et al., 2011) e foram inferiores às médias de P205; P365 e P550 (177,19kg; 236,68kg e $306,46 \mathrm{~kg}$, respectivamente) do 
arquivo de dados do controle de desempenho ponderal da Associação Brasileira dos Criadores de Zebu (ABCZ), dos Estados de TO, PA, Amazonas, Roraima, RO, AC e Amapá (SANTOS et al., 2012).

Nas características de peso, observaramse maiores médias dos machos em relação às fêmeas, sendo estas $6,72 \%$; $8,72 \%$ e $13,87 \%$ inferiores para P120; P210 e P450, respectivamente. Resultado semelhante ao encontrado por Silveira et al. (2004) e Toral et al. (2004) em bovinos Nelore do Mato Grosso do Sul, em que as fêmeas foram inferiores aos machos, em média, $8 \%$ para peso aos 205 dias, $11 \%$ para peso aos 365 dias e $13 \%$ para peso aos 550 dias de idade. Sendo que o aumento das diferenças entre pesos corporais de machos e fêmeas provavelmente está relacionado às diferenças fisiológicas e hormonais, que se intensificam à medida que o animal atinge a puberdade e inicia a sua vida sexual.

Tabela 1. Descrição por sexo e no geral das características de crescimento e reprodução de animais Nelore criados na Amazônia Legal

\begin{tabular}{lcccccc}
\hline Característica & & $\mathrm{N}$ & Média & $\mathrm{D}-\mathrm{P}$ & Mínimo & Máximo \\
\hline \multirow{3}{*}{ P120 $(\mathrm{kg})$} & *Geral & 94822 & 123 & 19,0 & 46 & 227 \\
& $\mathrm{M}$ & 47463 & 127 & 18,8 & 66 & 180 \\
& $\mathrm{~F}$ & 46932 & 119 & 17,3 & 66 & 180 \\
\hline \multirow{3}{*}{ P210 $(\mathrm{kg})$} & Geral & 88176 & 180 & 28,1 & 70 & 359 \\
& $\mathrm{M}$ & 44195 & 187 & 27,6 & 95 & 264 \\
& $\mathrm{~F}$ & 43592 & 172 & 25,1 & 95 & 264 \\
P450 $(\mathrm{kg})$ & Geral & 63580 & 262 & 45,5 & 110 & 612 \\
& $\mathrm{M}$ & 31589 & 279 & 47,3 & 111 & 612 \\
\hline PE450 $(\mathrm{mm})$ & $\mathrm{F}$ & 31375 & 245 & 36,3 & 110 & 528 \\
\hline IPP $($ meses $)$ & $\mathrm{M}$ & 23901 & 227 & 28,4 & 140 & 315 \\
\hline $\mathrm{N}$ & $\mathrm{F}$ & 38376 & 37 & 4,7 & 23 & 49 \\
\hline
\end{tabular}

$\mathrm{N}=$ número de animais; D-P = Desvio-padrão; P120 = Peso aos 120 dias de idade; P210 = Peso aos 210 dias de idade; P450 = Peso aos 450 dias de idade; PE450 = perímetro escrotal aos 450 dias; IPP = idade ao primeiro parto; $\mathrm{M}=$ machos; $\mathrm{F}=$ fêmeas. *Geral refere-se às médias antes da consistência dos dados.

O perímetro escrotal calculado aos 450 dias de idade é um parâmetro de fácil avaliação, possui alta correlação genética com características de crescimento como peso e correlação positiva com a fertilidade das filhas, apesar de ter baixa correlação com os aspectos relacionados à qualidade do sêmen (LOPES et al., 2011). A média desta característica foi de $22,7 \pm 2,84 \mathrm{~cm}$, resultado semelhante à média do PMGRN - Nelore Brasil de $23 \mathrm{~cm}$ (LÔBO et al., 2011).

A idade ao primeiro parto é uma característica que pode promover uma diminuição no intervalo de gerações possibilitando maior intensidade de seleção nas fêmeas. Outra vantagem é a redução de categorias animais em desenvolvimento dentro da fazenda, com consequência no aumento da taxa de desfrute do rebanho. A média desta característica neste estudo foi de $37 \pm$ 4,7 meses, valor este semelhante ao encontrado na média do PMGRN Nelore Brasil de 37 meses (LÔBO et al., 2011) e aos valores relatados por Dias et al. (2004), Boligon et al. (2007) e Boligon et al. (2008) as quais foram 
de 35,$67 ; 36,00 ; 34,60 ; 36,21$ e 36,50 meses, respectivamente, para fêmeas da raça Nelore.

As estimativas dos componentes de (co)variância e dos parâmetros genéticos das análises entre o P120 e as outras características de peso dos dados da Amazônia Legal (Tabela 2) foram inferiores aos valores obtidos por Santos et al. (2012) para as estimativas de herdabilidade direta do P205, P365 e P550 (respectivamente iguais a 0,$33 ; 0,51$ e 0,41$)$ também para animais Nelore da região Amazônica. A diferença deveu-se, possivelmente, à base genética, aos modelos e aos rebanhos estudados distintos.

Tabela 2. Estimativas dos componentes de (co)variância e dos parâmetros genéticos provenientes das análises conjuntas de duas características, entre o peso aos 120 dias de idade - P120 (variável 1) e outras características (variável 2), dos dados da Amazônia Legal

\begin{tabular}{crrr}
\hline \multirow{2}{*}{ Parâmetro genético* } & \multicolumn{3}{c}{ Peso aos 120 dias de idade (P120) - (1) } \\
\cline { 2 - 4 } & $\mathrm{P} 210(2)$ & $\mathrm{P} 450(2)$ & $\mathrm{PE} 450(2)$ \\
\hline$\sigma_{\mathrm{A} 1}{ }^{2}$ & 34,14 & 88,74 & 42,95 \\
$\sigma_{\mathrm{A} 1,2}$ & 45,99 & 145,82 & 42,19 \\
$\sigma^{2}{ }_{\mathrm{A} 2}$ & 73,91 & 287,75 & 200,40 \\
$\sigma_{\mathrm{E} 1}$ & 93,64 & 79,00 & 90,68 \\
$\sigma_{\mathrm{E} 1,2}$ & 99,51 & 66,25 & 36,13 \\
$\sigma_{\mathrm{E} 2}^{2}$ & 178,25 & 316,41 & 248,37 \\
$\sigma_{\mathrm{P} 1}^{2}$ & 174,54 & 182,76 & 174,06 \\
$\sigma_{\mathrm{P} 1,2}$ & 211,30 & 212,06 & 78,32 \\
$\sigma_{\mathrm{P} 2}$ & 345,98 & 604,16 & 448,77 \\
$\mathrm{~h}^{2}{ }_{\mathrm{d} 1}$ & 0,20 & 0,49 & 0,25 \\
$\mathrm{~h}^{2}{ }_{\mathrm{d} 2}$ & 0,21 & 0,48 & 0,45 \\
$\mathrm{~h}^{2}{ }_{\mathrm{m} 1}$ & 0,08 & 0,23 \\
$\mathrm{~h}^{2}{ }_{\mathrm{m} 2}$ & 0,27 & - & - \\
$\mathrm{r}_{\mathrm{A}}$ & 0,27 & 0,91 & 0,45 \\
$\mathrm{e}^{2}{ }_{1}$ & 0,92 & 0,43 & 0,52 \\
$\mathrm{e}^{2}{ }_{2}$ & 0,54 & 0,52 & 0,55 \\
\hline
\end{tabular}

P210 = peso aos 210 dias de idade $(\mathrm{kg}) ; \mathrm{P} 450=$ peso aos 450 dias de idade $(\mathrm{kg})$; PE450 = perímetro escrotal aos 450 dias de idade $(\mathrm{mm}) .{ }^{*} \sigma_{\mathrm{Ai}, \mathrm{j}}=(\mathrm{co})$ variância genética aditiva; $\sigma_{\mathrm{Ei}, \mathrm{j}}=(\mathrm{co})$ variância residual; $\sigma_{\mathrm{P}_{\mathrm{i}, \mathrm{j}}}=\left(\right.$ co)variância fenotípica; $\mathrm{h}_{\mathrm{di}}^{2}=$ herdabilidade direta; $\mathrm{h}_{\mathrm{mi}}^{2}=$ herdabilidade materna; $\mathrm{r}_{\mathrm{A}}=$ correlação genética; $\mathrm{e}_{\mathrm{i}}^{2}=$ fração da variância fenotípica devida ao efeito de ambiente temporário. Para as variâncias, $\mathrm{i}=\mathrm{j}$; para as covariâncias $\mathrm{i} \neq \mathrm{j} ; \mathrm{i}, \mathrm{j}=1,2$.

A idade ao primeiro parto é uma característica que tem grande importância zootécnica, pois marca o início da vida produtiva das fêmeas. Nos Estados do Norte e Nordeste, a IPP pode ser alta, segundo Azêvedo et al. (2006), se for considerado apenas o peso ideal das novilhas à época de entrada em reprodução, dadas as características peculiares dessas regiões. A herdabilidade da IPP teve valor mediano $(0,21 \pm 0,03)$, ainda que a maioria das características reprodutivas apresente estimativas de herdabilidade de baixa magnitude, segundo Boligon et al. (2007). O valor da estimativa de herdabilidade obtido neste estudo foi superior ao valor encontrado por Dias et al. (2004) com média de 
0,16 , e inferior ao relatado por Bertazzo et al. (2004), que encontraram herdabilidade de 0,37. Dias et al. (2004) estudaram apenas dados de um rebanho no Estado de São Paulo com manejo diferenciado, enquanto que Bertazzo et al. (2004) estudaram dados históricos nacionais da Associação Brasileira de Criadores de Zebu (ABCZ), o que não permite maiores inferências pelas diferenças nas bases de dados estudadas, modelos e programas aplicados.

Comparando as herdabilidades das características dos dados obtidos na Amazônia Legal com os dados do PMGRN - Nelore Brasil (Tabela 3), pode ser observado que as características tiveram resultados divergentes entre os dois bancos de dados, indicando a presença de IGA, especialmente em relação aos aspectos maternos, de peso ao sobreano e de IPP. Toral et al. (2004) estudaram o efeito da IGA sobre as características de crescimento de bovinos da raça Nelore criados em três microrregiões homogêneas do estado do Mato Grosso do Sul, e concluíram que o efeito das microrregiões sobre os pesos estudados evidencia as particularidades de cada uma. Com isso, a consideração da IGA nos processos de avaliação genética, principalmente em programas de alcance nacional, faz-se necessária.

Tabela 3. Estimativas dos componentes de (co)variância e dos parâmetros genéticos provenientes das análises conjuntas de duas características, entre o peso aos 120 dias de idade - P120 (variável 1) e outras características (variável 2), na base geral do PMGRN - Nelore Brasil

\begin{tabular}{|c|c|c|}
\hline \multirow{2}{*}{ Parâmetro genético* } & \multicolumn{2}{|c|}{ Peso aos 120 dias de idade (P120) - (1) } \\
\hline & P450 (2) & PE450 (2) \\
\hline$\sigma_{\mathrm{A} 1}^{2}$ & 42,37 & 42,37 \\
\hline$\sigma_{\mathrm{A} 1,2}$ & 79,08 & 2,89 \\
\hline$\sigma_{\mathrm{A} 2}^{2}$ & 217,16 & 2,16 \\
\hline$\sigma_{\mathrm{m} 1}^{2}$ & 19,29 & 19,29 \\
\hline$\sigma_{\mathrm{m} 1, \mathrm{~m} 2}$ & - & - \\
\hline$\sigma_{\mathrm{m} 2}^{2}$ & - & - \\
\hline$\sigma_{\mathrm{E} 1}^{2}$ & 91,40 & 91,40 \\
\hline$\sigma_{\mathrm{E} 1,2}$ & 84,05 & 3,77 \\
\hline$\sigma_{\mathrm{E} 2}^{2}$ & 361,20 & 2,61 \\
\hline$h_{d 1}^{2}$ & 0,23 & 0,23 \\
\hline $\mathrm{h}_{\mathrm{d} 2}^{2}$ & 0,34 & 0,43 \\
\hline $\mathrm{h}_{\mathrm{ml}}^{2}$ & 0,11 & 0,11 \\
\hline $\mathrm{h}_{\mathrm{m} 2}^{2}$ & - & - \\
\hline$r_{A}$ & 0,82 & 0,30 \\
\hline
\end{tabular}

As correlações de rank entre DEPs das características estudadas obtidas nos arquivos da base geral do PMGRN Nelore Brasil e nos registros dos animais criados na região da Amazônia Legal foram as seguintes: 0,56 para DP120m, 0,77 para DP120d, 0,58 para DP210m, 0,79 para DP210d, 0,83 para 
DP450, 0,79 para DPE450 e 0,38 para DIPP (sendo, DP120m = DEP materna para peso aos 120 dias de idade; DP120d = DEP direta para peso aos 120 dias de idade; DP210m = DEP materna para peso aos 210 dias de idade; DP210d = DEP direta para peso aos 210 dias de idade; DP450 = DEP para peso aos 450 dias de idade; DPE450 = DEP para perímetro escrotal aos 450 dias de idade; DIPP = DEP para idade ao primeiro parto).

Os coeficientes de correlação de Spearman entre as DEPs para DP120m, DP210m e DIPP foram de baixa magnitude, sugerindo que há mudança na classificação dos animais testados na região da Amazônia Legal em relação aos à base geral do PMGRN - Nelore Brasil. Como houve mudança na classificação dos animais para as características citadas acima, há indícios de IGA, segundo Robertson (1959), o qual determinou que, se correlações genéticas para a mesma característica em diferentes ambientes fossem menores que 0,80 evidenciariam a existência de IGA.

A alteração da classificação de touros também foi observada por Toral et al. (2004) para as características peso ao nascimento, aos 205, 365 e 550 dias, em bovinos Nelore provenientes de três regiões de Mato Grosso do Sul. Lopes et al. (2008) observaram baixas correlações entre as DEPs de animais avaliados em diferentes ambientes na região Sul do Brasil, para peso ao nascimento, peso à desmama e peso ao sobreano na raça Nelore. Faria et al. (2011) não evidenciaram efeito de interação genótipo-ambiente sobre $o$ peso à desmama em animais Nelore de seis Estados brasileiros. Ribeiro et al. (2009), estudando o efeito de IGA em dados de animais Nelore de São Paulo e Mato Grosso do Sul, também não evidenciaram efeito significativo para a característica de peso à desmama. $\mathrm{O}$ efeito de IGA, portanto, parece mais evidente para pesos e características pós-desmama, como constatado para P450 em seis Estados do Sul, CentroOeste e Sudeste, por Solar Diaz et al. (2011), os quais recomendaram a inclusão deste efeito na avaliação genética da característica, e por Guidolin et al. (2012) em outros seis Estados, também para a raça Nelore, o que corrobora com o presente estudo nos resultados obtidos para as estimativas de herdabilidades.

As análises da interação genótipoambiente, por meio das estimativas de herdabilidade, evidenciaram maiores efeitos sobre os aspectos maternos, de peso aos 450 dias de idade e idade ao primeiro parto, enquanto que as correlações entre classificações mostraram fortes evidências em quase todas as características estudadas.

\section{AGRADECIMENTOS}

À CAPES pelas Bolsas de estudos concedidas. $\grave{A}$ ANCP pela concessão dos dados do PMGRN Nelore Brasil.

\section{REFERÊNCIAS}

\author{
AZEVÊDO, D.M.M.R.; MARTINS \\ FILHO, R.; LOBO, R.N.B.; \\ MALHADO, C.H.M.; LÔBO, R.B.; \\ MOURA, A.A.A.; PIMENTA FILHO, \\ E.C. Desempenho reprodutivo de vacas \\ Nelore no Norte e Nordeste do Brasil.
}

Revista Brasileira de Zootecnia, v.35, n.3, p.988-996, 2006.

BASU, S.B.; CHATTARAJI, J. Genotype-environment interaction in the evaluation of sires of crossbred cows. Indian Journal of Animal Science, v.58, n.7, p.808-811, 1988. 
Rev. Bras. Saúde Prod. Anim., Salvador, v.14, n.3, p.599-608 jul./set., 2013 http://www.rbspa.ufba.br ISSN 15199940

BERTAZZO, R.P.; FREITAS, R.T.F.; GONÇALVES, T.M.; PEREIRA, I.G.; ELER, J.P.; FERRAZ, J.B.S.;

OLIVEIRA, A.I.G.; ANDRADE, I.F. Parâmetros genéticos de longevidade e produtividade de fêmeas da raça Nelore. Revista Brasileira de Zootecnia, v.33, p.1118-1127, 2004.

BOLDMAN, K.G.; KRIESE, L.A.; VAN VLECK, L.D.; Van TASSELL, S.D.; KACHMAN, S.D. A manual for use of MTDFREML: a set of programs to obtain estimates of variance and covariance. [DRAFT]. Lincoln: Department of Agriculture, Agricultural Research Service, 1995. 120p.

BOLIGON, A.A.; RORATO, P.R.N.; ALBUQUERQUE, L.G. Correlações genéticas entre medidas de perímetro escrotal e características produtivas e reprodutivas de fêmeas da raça Nelore. Revista Brasileira de Zootecnia, v.36, p.565-571, 2007.

BOLIGON, A.A.; VOZZI, P.A.; NOMELINI, J.; RORATO, P.R.N.; BEZERRA, L.A.F.; LÔBO, R.B. Parâmetros genéticos para idade ao primeiro parto estimados por diferentes modelos para rebanhos da raça Nelore. Ciência Rural, v.38, p.432-436, 2008.

CORREA, M.B.B.; DIONELLO, N.J.L.; CARDOSO, F.F. Caracterização da interação genótipo-ambiente $\mathrm{e}$ comparação entre modelos para ajuste do ganho pós-demama de bovinos Devon via normas de reação. Revista Brasileira de Zootecnia, v.38, n.8, p.1460-1467, 2009.

DIAS, L.T.; FARO, L.; ALBUQUERQUE, L.G. Estimativa de herdabilidade para idade ao primeiro parto de novilhas da raça Nelore. Revista Brasileira de Zootecnia, v.33, p.97-102, 2004.
DIMOV, G.; ALBUQUERQUE, L.G.; KEOWN, J.F.; VAN VLECK, L.D.; NORMAN, H.D. Variance of interaction effects of sire and herd for yield traits of Holsteins in California, New York, and Pennsylvania with an animal model. Journal of Dairy Science, v.78, n.4, p.939-946, 1995.

FALCÃO, A.J.S.; MARTINS, E.N.; COSTA, C.N.; SAKAGUTI, E.S.; MAZUCHELI, J. Heterocedasticidade entre Estados para produção de leite em vacas da raça Holandesa, usando métodos Bayesianos via amostrador de Gibbs.

Revista Brasileira de Zootecnia, v.35, n.2, p.405-414, 2006.

FARIA, C. de U.; TERRA, J.P.; YOKOO, M.J.; MAGNABOSCO, C. de U.; ALBUQUERQUE, L.G.; LÔBO, R.B. Interação genótipo-ambiente na análise genética do peso ao desmame de bovinos Nelore sob enfoque bayesiano.

Acta Scientiarum. Animal Sciences, v.33, n.2, p.213-218, 2011.

GUIDOLIN, D.G.F.; BUZANSKAS, M.E.; RAMOS, S.B.; VENTURINI, G.C.; LÔBO, R.B.; PAZ, C.C.P.; MUNARI, D.P.; OLIVEIRA, J.A. Genotype-environment interaction for post-weaning traits in Nellore beef cattle. Animal Production Science, v.52, n.11, p. 975-980, 2012.

INSTITUTO BRASILEIRO DE GEOGRAFIA E ESTATÍSTICA - IBGE. Censo Agropecuário 2006. 2009.

Disponível em:

$<$ http://www.ibge.gov.br/home/estatistica/ economia/agropecuaria/censoagro/brasil_ 2006/Brasil_censoagro2006.pdf $>$. Acesso em: 03 out. 2013.

KOLMODIN, R.; BIJMA, P. Response to mass selection when the genotype by environment interaction is modelled as a 
Rev. Bras. Saúde Prod. Anim., Salvador, v.14, n.3, p.599-608 jul./set., 2013 http://www.rbspa.ufba.br ISSN 15199940

linear reaction norm. Genetics, Selection, Evolution, v.36, p.435-454, 2004.

LÔBO, R.B.; BEZERRA, L.A.F.; MAGNABOSCO, C. de U.;

ALBUQUERQUE, L.G.; SAINZ, R.D.; BERGMANN, J.A.G.; FARIA, C. de U., OLIVEIRA, H.N. Avaliação genética de touros das raças Nelore, Guzerá, Brahman e Tabapuã:

Sumário 2011. Ribeirão Preto, SP: ANCP, 2011. 136p.

LOPES, D.T.; VIU, M.A.O.; MAGNABOSCO, C.U.; FARIA, C.U.; FERRAZ, H.T.; TROVO, J.B.F.; TERRA, J.P.; PIRES, B.C. Estimativa de parâmetros genéticos de características andrológicas de touros jovens da raça Nelore por meio da inferência bayesiana. Revista Brasileira de Saúde e Produção Animal [online], v.12, n.1, p.72-83, 2011.

LOPES, J.S.; RORATO, P.R.N.; WEBER, T.; BOLIGON, A.A.; COMIN, J.G.; DORNELLES, M.A. Efeito da interação genótipo $\mathrm{x}$ ambiente sobre o peso ao nascimento,/ aos 205 e aos 550 dias de idade de bovinos da raça Nelore na Região Sul do Brasil. Revista Brasileira de Zootecnia, v.37, p.54-60, 2008.

RIBEIRO, S.; ELER, J.P.; BALIEIRO, J.C.C.; FERRAZ, J.B.S.; PEDROSA, V.B.; MATTOS, E.C. Influência da interação genótipo $\mathrm{x}$ ambiente sobre $\mathrm{o}$ peso à desmama em bovinos da raça Nelore. Arquivo Brasileiro de Medicina Veterinária e Zootecnia, v.61, n.3, p.668-675, 2009.

ROBERTSON, A. The sampling variance of genetic correlation coefficient. Biometrics, v.15, p.469485, 1959.
SANTOS, G.C.J.; LOPES, F.B.; MARQUES, E.G.; SILVA, M.C.; CAVALCANTE, T.V.; FERREIRA, J.L. Tendência genética para pesos padronizados aos 205, 365 e 550 dias de idade de bovinos Nelore da região norte do Brasil. Acta Scientiarum. Animal Sciences, v.34, n.1, p.97-101, 2012.

SAS Institute. SAS Users guide: statistics. Version 9.1. Cary, NC, 2002.

SILVEIRA, J.C.; McMANUS, C.; MASCIOLI, A,S.; SILVA, L.O.C.; SILVEIRA, A.C.; GARCIA, J.A.S.; LOUVANDINI, H. Fatores ambientais e parâmetros genéticos para características produtivas e reprodutivas em um rebanho Nelore no Estado do Mato Grosso do Sul. Revista Brasileira de Zootecnia, v.33, p.1432-1444, 2004.

SOLAR DIAZ, I.P.; OLIVEIRA, H.N.; BEZERRA, L.A.F.; LÔBO, R.B.

Genotype by environment interaction in Nellore cattle from five Brazilian states. Genetics and Molecular Biology, v.34, n.3, p.435-442, 2011.

\section{SUPERINTENDÊNCIA DO DESENVOLVIMENTO DA AMAZÔNIA - SUDAM. Amazônia}

Legal. 2013. Disponível em: $<$ http://www.sudam.gov.br/amazonialegal>. Acesso em: 03 out. 2013.

TORAL, F.L.B.; SILVA, L.O.C.; MARTINS, E.M.; GONDO, A.; SIMONELLI, S.M. Interação genótipo $\mathrm{x}$ ambiente em características de crescimento em bovinos da raça Nelore no Mato Grosso do Sul. Revista Brasileira de Zootecnia, v.33, p.14451455, 2004.

Data de recebimento: 11/03/2013

Data de aprovação: 27/09/2013 